\title{
Postmortem study of the spinal cord showing snake-eyes appearance due to damage by ossification of the posterior longitudinal ligament and kyphotic deformity
}

\author{
J Mizuno*,1, H Nakagawa $^{1}$, H-S Chang ${ }^{1}$ and Y Hashizume ${ }^{2}$ \\ ${ }^{1}$ Department of Neurological Surgery, Aichi Medical University, Aichi, Japan; ${ }^{2}$ Institute of Medical Science for Aging, \\ Aichi Medical University, Aichi, Japan
}

\begin{abstract}
Clinical design: A case report.
Objectives: To elucidate the clinical role of snake-eyes appearance in this case, correlation between radiological, clinical and postmortem study was performed.

Setting: Aichi, Japan.

Case report: A 73-year-old man developed weakness and pain in the upper limbs due to kyphotic deformity secondary to laminectomy for cervical ossification of the posterior longitudinal ligament. Axial magnetic resonance imaging revealed snake-eyes appearance from C4 to C6. He died of acute myocardial infarction 3 months after anterior decompressive surgery.

Results: A postmortem examination of the cervical spinal cord showed small cystic six necrotic areas at the junction of the central gray matter and the ventrolateral posterior column, one in the right and one in the left, in association with neuronal loss in the anterior horn.

Conclusions: Bilateral small intramedullary high-signal areas known as 'snake-eyes appearance' located around the central gray matter and the ventrolateral posterior column, are associated with neuronal loss in the compressed anterior horn that played an important role in worsening weakness of the upper limbs.
\end{abstract}

Spinal Cord (2005) 43, 503-507. doi:10.1038/sj.sc.3101727; published online 8 March 2005

Keywords: snake-eyes appearance; compression myelopathy; magnetic resonance imaging; cystic necrosis; neuronal loss

\begin{abstract}
Introduction
Intramedullary high-signal intensity (IMHSI) is a wellknown radiological finding of magnetic resonance (MR) imaging. Snake-eyes appearance (SEA), defined as small high-signal areas around the central gray matter and the ventrolateral posterior column, one in the right and one in the left, is a unique IMHSI of the spinal cord with compression myelopathy. ${ }^{1-5}$ Although this phenomenon is commonly observed in cases with cervical spondylosis (CS) or ossification of the posterior longitudinal ligament (OPLL), it's clinical role is obscure. Seemingly, the controversy over the prognostic value of SEA is derived from the lack of clinicopathological correlations with small high-signal lesions. Thus, it is important to examine the pathological changes of the compressed cervical spinal cord with SEA confirmed by MR imaging in relation to the clinical manifestations.
\end{abstract}

*Correspondence: J Mizuno, Department of Neurological Surgery, Aichi Medical University, 21 Karimata Yazako Nagakute, Aichi-gun, Aichi 480-1195, Japan
We report an autopsy study of SEA with weakness and pain of the upper limbs caused by canal stenosis secondary to OPLL and postlaminectomy kyphotic deformity of the cervical spine.

\section{Case report}

A 73-year-old man was admitted to hospital with weakness and pain of the upper limbs. He had undergone laminoplasty for cervical OPLL 6 years previously. Neurological examination showed moderate weakness of the deltoid, biceps, triceps and grip strength with muscle atrophy, hyperreflexia in the knee and ankle jerks, hypesthesia in C5-T1 dermatome, decreased position sensation on knees and toes and severe gait difficulty. Fasciculation of the upper limbs was not observed. Cervical radiograph revealed kyphotic deformity secondary to the previously performed open-door laminoplasty from C3 to C7 for OPLL (Figure 1). Sagittal T2-weighted MR imaging revealed severe cord 
compression with linear IMHSI from C4 to C6. Axial T2-weighted MR imaging revealed SEA consecutively from C4 to C6 (Figure 2). Anterior decompression and fixation with an interbody cage (Pyramesh, Medtronics

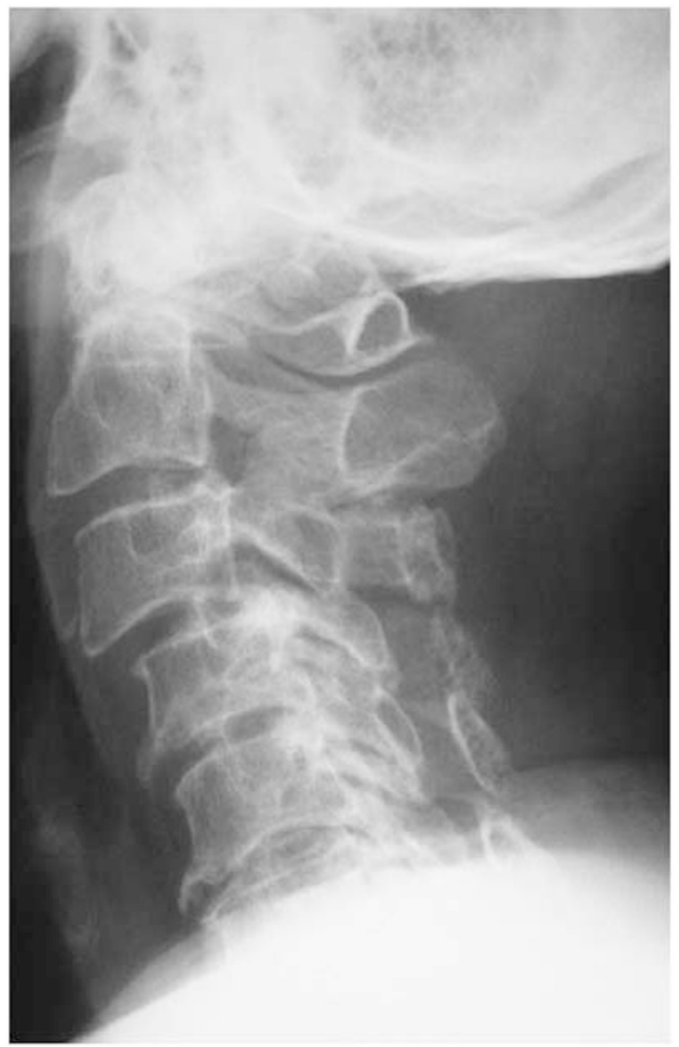

Figure 1 Cervical radiograph showing kyphotic deformity with subluxation at $\mathrm{C} 3 / 4$
Sofamor Danek, Tennessee, USA) packed with bone chips collected from the resected vertebral bodies and an anterior plate (ORION, Medtronics Sofamor Danek, Tennessee, USA) were performed (Figure 3). Postoperatively, his neck and arm pain subsided with medication, although his motor weakness remained without significant improvement. His neurological status was relatively stable; however, he died

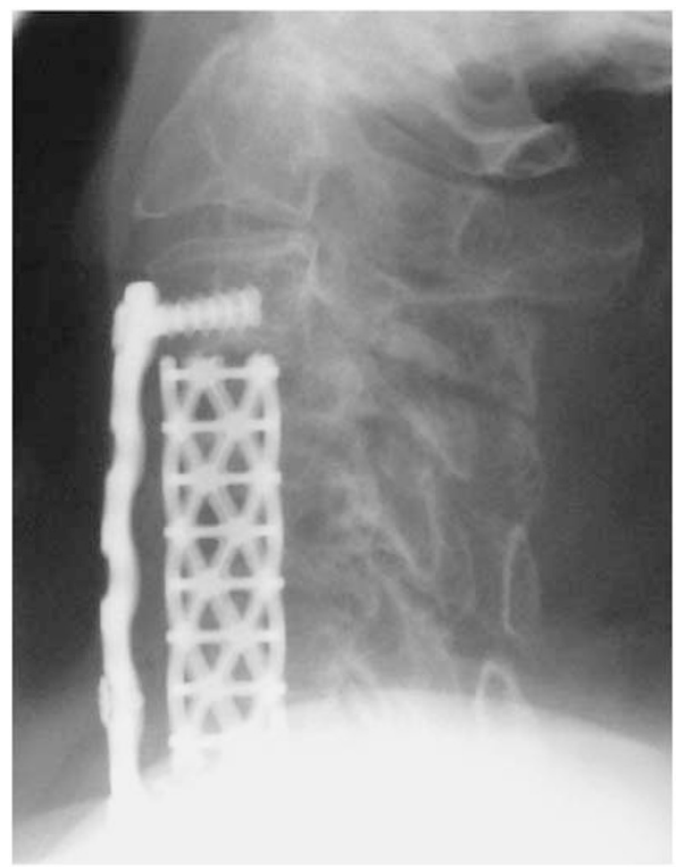

Figure 3 Postoperative cervical radiograph showing restoration of normal cervical spinal alignment with interbody Pyramesh and Orion plate

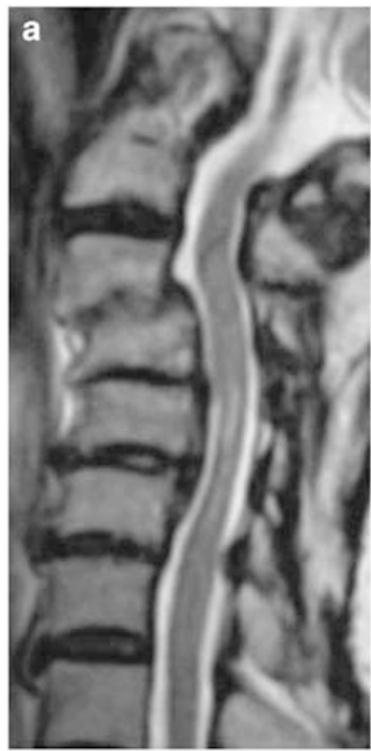

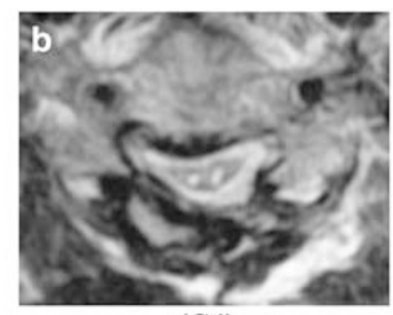

(C4)

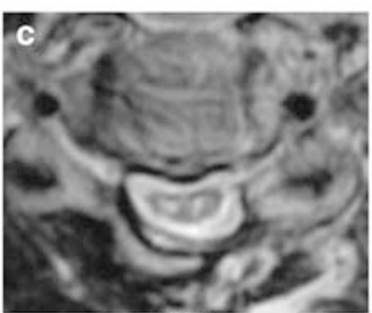

(C5)

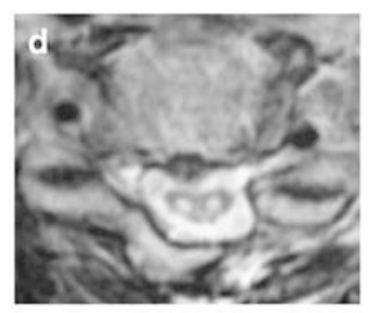

(C6)

Figure 2 (a) Sagittal T2-weighted MR imaging revealing linear IMHSI from C4 to C6 with posterior displacement of the cervical spinal cord. (b-d) Axial T2-weighted MR imaging revealing atrophic cervical cord with SEA at C4-C6 


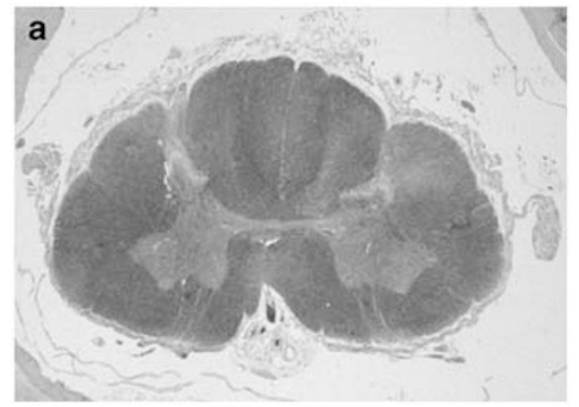

(C3)

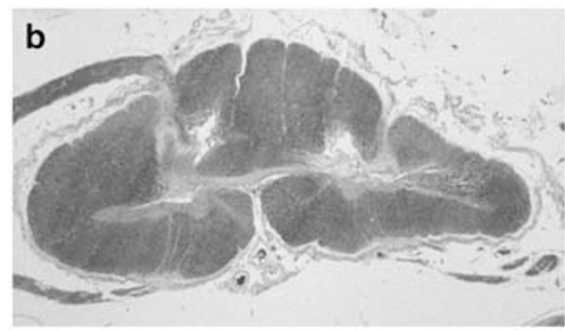

(C4)

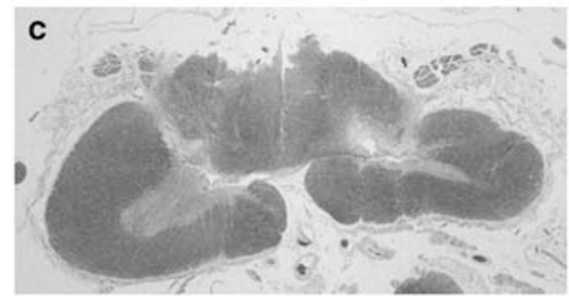

(C5)

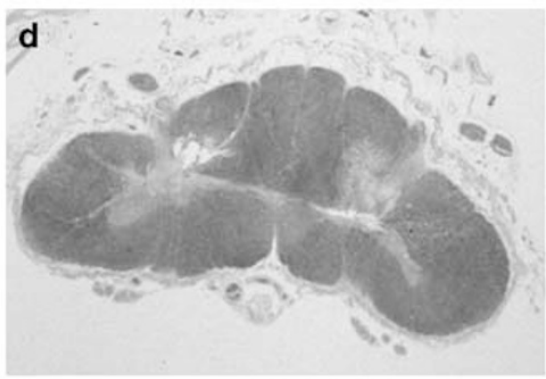

(C6)

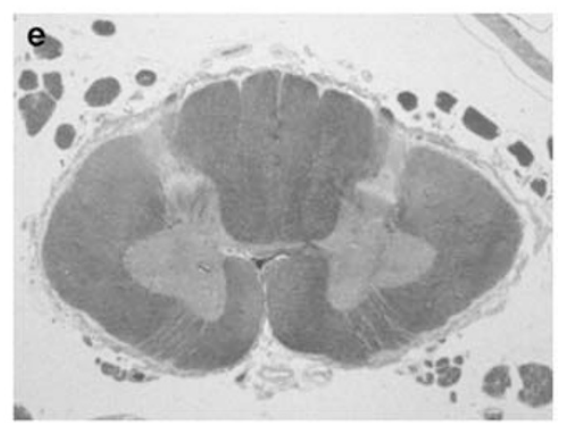

(C7)

Figure 4 Photomicrograph of the transverse cervical spinal cord. (a) C3, (b) C4, (c) C5, (d) C6, (e) C7; C3 and C7 cords were relatively well preserved, although C4-C6 showed intramedullary cystic necrosis around the central gray matter and the ventrolateral posterior column (original magnification $\times 15, \mathrm{~K}-\mathrm{B}$ stain)

suddenly of acute myocardial infarction 3 months after operation.

Postmortem study of the cervical spinal cord showed severe cord deformity with atrophy from C4 to C6, although good surgical decompression had been performed. Hematoxylin-eosin (H-E) stain and KluverBarrera $(\mathrm{K}-\mathrm{B})$ stain were applied. Flattening of the spinal cord was slightly asymmetric, with a right-side predominance. Cystic cavitation with surrounding spongy state was observed bilaterally at the junction of the central gray matter and the ventrolateral posterior column from $\mathrm{C} 4$ to $\mathrm{C} 6$. There was a sizable discrepancy regarding the degree of deformity between the spinal cord and the anterior horn of the left-sided C4, C5 and C6 and the right-sided C5. At these levels, the anterior horn was severely flattened despite mild-to-moderate cord compression (Figure 4). There was a significant neuronal loss in the compressed anterior horn except for the right-sided C5 (Figure 5). The fibrous thickening of the small veins with narrowing of the lumen around the cavity was prominent, although reactive gliosis was not prominent (Figure 6).

\section{Discussion}

There have been many investigations into whether or not IMHSI in patients with compression myelopathy acts as a poor prognostic indicator. ${ }^{1-8}$ SEA is a unique radiological finding that can be observed in cases with compression myelopathy, kyphotic deformity or spinal cord injury. The term 'snake-eyes appearance' is derived from the resemblance of the bilateral small IMHSI in the compressed cord to the face of a snake. This term may be the same as 'fried-egg appearance' demonstrated by computed-tomographic myelography by Iwasaki et $a{ }^{9}{ }^{9}$ Some authors suggested that SEA is an irreversible change in the spinal cord, like cystic necrosis. ${ }^{3,7,8,10}$ Others suggested that it is a reversible change, similar to edema. ${ }^{3,6,8}$ This confusion arises from a lack of precise pathological correlation of the compressed spinal cord with SEA.

In the present case, we attempted to correlate the neurological presentation with MR findings, and compared the results of precise pathological examination. Sagittal MR imaging showed multisegmental linear 

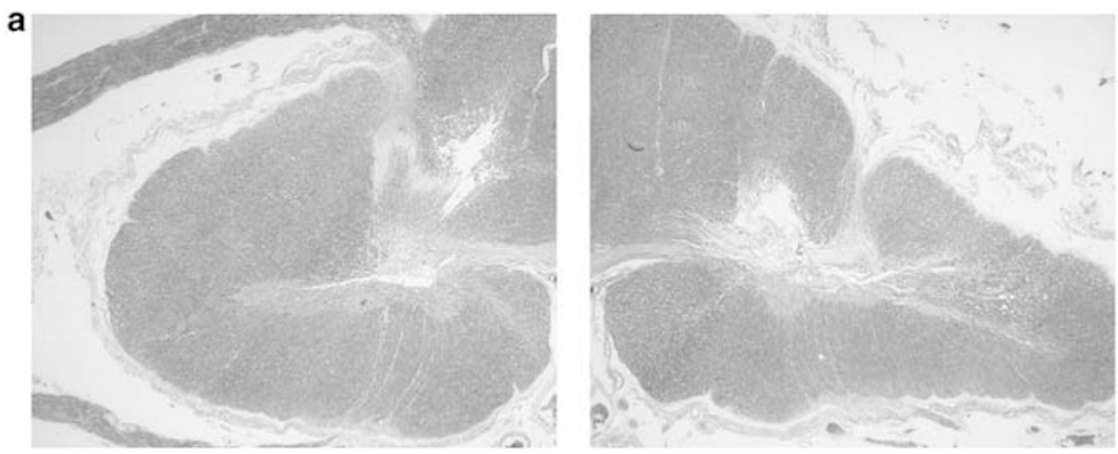

(C4)
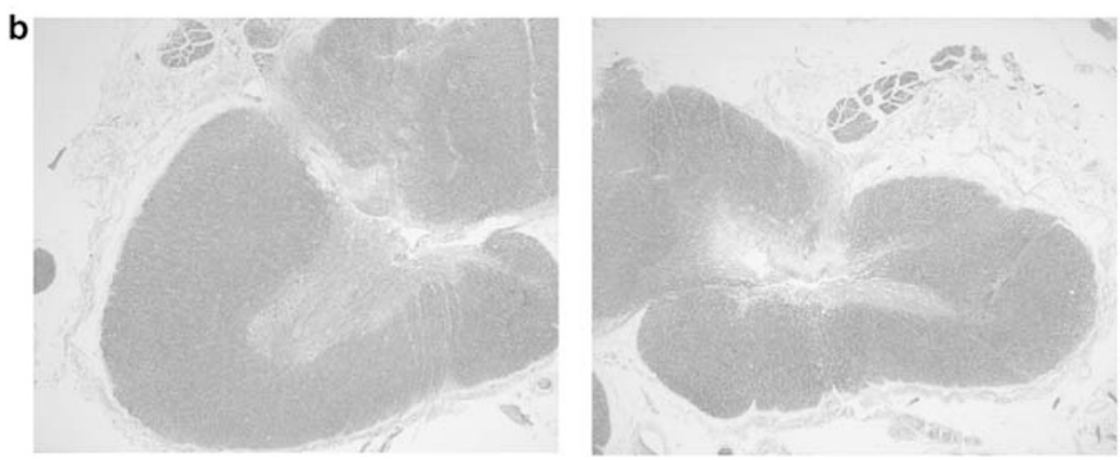

(C5)
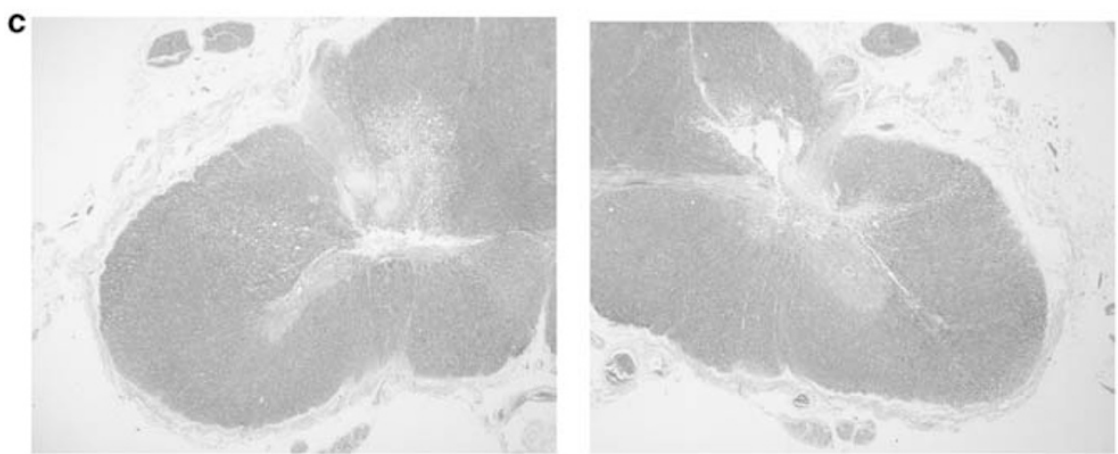

(C6)

Figure 5 Higher power of photomicrographs at C4 (a), C5 (b) and C6 (c) showed severe flattening of the anterior horn with neuronal loss associated with intramedullary cystic necrosis (original magnification $\times 40, \mathrm{~K}-\mathrm{B}$ stain)

IMHSI from $\mathrm{C} 4$ to $\mathrm{C} 6$ and axial MR imaging revealed SEA, confirming that this linear IMHSI was correlated with SEA. In addition to the cord compression with posterior shifting, atrophy of the spinal cord was apparent because of the relatively wide subarachnoid space. Postmortem study of the spinal cord showed formation of small cavities at the junction of the central gray matter and the ventrolateral posterior column with surrounding spongy state from $\mathrm{C} 4$ to $\mathrm{C} 6$, bilaterally. This pathological finding correlated well with the highsignal intensity observed in T2-weighted MR imaging. The authors believe that SEA may indicate an irreversible change caused by cystic cavity formation, not a reversible change as gliosis or edema.
The majority of previous investigators have described the possible pathology or the morphological characteristics of the SEA without examining the precise pathological changes of the whole transverse spinal cord. We found that the neurons in the flattened anterior horn remarkably reduced at the levels of the intramedullary cavity formation. Segmental motor weakness in our patient with atrophy of the upper limbs may be caused by neuronal loss in the anterior horn, while segmental sensory deficits could be explained by the formation of a cystic cavity at the junction of the central gray matter and the ventrolateral posterior column.

Pathogenesis of SEA is still obscure. Long-term cord compression and secondary vascular changes are 


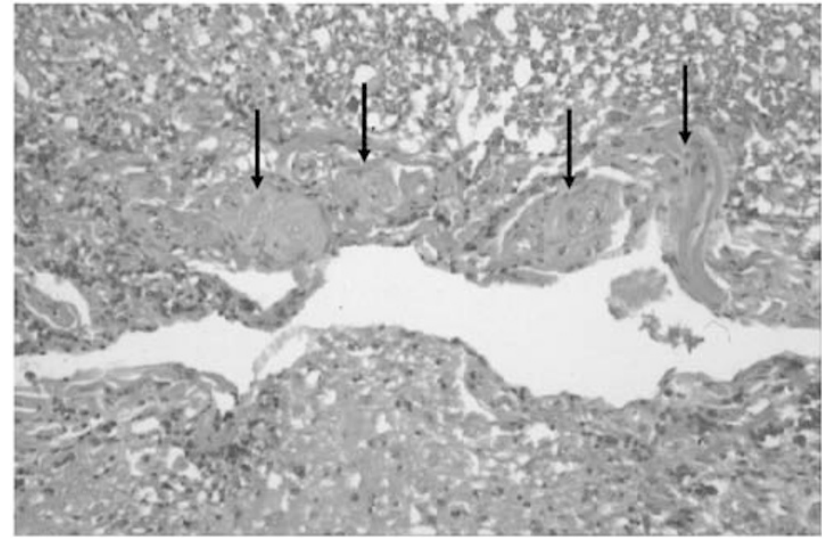

Figure 6 Photomicrograph showing fibrous thickening of the small veins with narrowing of the lumen (arrow) around cysts (original magnification $\times 100, \mathrm{H}-\mathrm{E}$ stain)

considered to be possible mechanisms. ${ }^{11-14}$ The fibrous thickening of small veins with narrowing of the lumen around the cysts in this case may indicate that venous infarction plays an important role in the formation of cystic necrosis. Although MR imaging revealed SEA at the central gray matter and the ventrolateral posterior column, damage of anterior horn cells should be considered as a causative lesion for motor dysfunction of the upper limbs.

\section{Conclusion}

From observation of this case, SEA on T2-weighted MR imaging may visualize the small intramedullary cysts due to venous infarction. Associated neuronal loss in the anterior horn could be responsible for segmental weakness and atrophy of the upper limbs. Accumulation of precise pathological analyses of SEA is essential in order to elucidate the pathophysiology of the intramedullary cystic necrosis in compression myelopathy.

\section{References}

1 Mehalic TF, Pezzuti RT, Applebaum BI. Magnetic resonance imaging and cervical spondylotic myelopathy. Neurosurgery 1990; 26: 217.

2 Ramanauskas WL et al. MR imaging of compressive myelomalacia. J Comput Assist Tomogr 1989; 13: 399.

3 Takahashi $\mathrm{M}$ et al. Chronic cervical cord compression: clinical significance of increased intensity on MR images. Radiology 1989; 173: 219-224.

4 Yone $\mathrm{K}$ et al. Preoperative and postoperative magnetic resonance image evaluations of the spinal cord in cervical myelopathy. Spine 1992; 17: S388-S392.

5 Mizuno $\mathbf{J}$ et al. Surgical outcomes analysis of cervical OPLL and spondylosis patients with intrinsic high signal intensity lesions on MRI. Prog Comput Imaging 1999; 21: $5-11$.

6 Wada E et al. Intramedullary changes of the spinal cord in cervical spondylotic myelopathy. Spine 1995; 20: 2226-2332.

7 Al-Mefty O et al. Myelopathic cervical spondylotic lesions demonstrated by magnetic resonance imaging. J Neurosurg 1988; 68: 217-222.

8 Matsuda $\mathrm{Y}$ et al. Increased MR signal intensity due to cervical meylopathy. J Neurosurg 1991; 74: 887-892.

9 Iwasaki Y et al. CT myelography with intramedullary enhancement in cervical spondylosis. J Neurosurg 1985; 63: 363-366.

10 Okada Y et al. Magnetic resonance imaging study on the results of surgery for cervical compression myelopathy. Spine 1993; 18: 2024-2029.

11 Bedford PD, Bosanquet FD, Russell WR. Degeneration of the spinal cord associated with cervical spondylosis. Lancet 1952; 2: 55-59.

12 Mair WGP, Druckman R. The pathology of spinal cord lesions and their relation to the clinical features in protrusions of cervical intervertebral discs. Brain 1953; 76: 70-91.

13 Ono K et al. Cervical myelopathy secondary to multiple spondylotic protrusions: a clinicopathological study. Spine 1977; 2: 109-125.

14 Murakami N, Muroga T, Sobue I. Cervical myelopathy due to ossification of the posterior longitudinal ligament: a clinicopathologic study. Arch Neurol 1978; 35: 33-36. 